'Departamento de Salud Pública, Facultad de Medicina Universidad de Concepción. 2Departamento de Educación Médica, Facultad de Medicina, Universidad de Concepción. ${ }_{3}^{3}$ Programa de Estudios sobre la Responsabilidad Social,

Universidad de Concepción aPsicólogo, Magíster en Psicología con mención en Psicología Educativa.

bPsicóloga Educacional, Doctora en Educación.

Recibido el 19 de mayo de 2013, aceptado el 17 de diciembre de 2013

Correspondencia a: Débora Alvarado $\mathrm{F}$

Departamento de Salud

Pública, Facultad de Medicina,

Universidad de Concepción. Janequeo s/n, Barrio Universitario, Concepción, Chile.

Teléfono: 56-41-2204924 dalvarado@udec.c

\section{Construcción y análisis psicométrico de un cuestionario para evaluar el comportamiento médico socialmente responsable}

\author{
DÉBORA ALVARADO F. ${ }^{1}$, CRISTHIAN PÉREZ V., ${ }^{2, a}$, GRACIA NAVARRO S. ${ }^{3, b}$
}

\begin{abstract}
Background: Professional Social Responsibility is now imperative for the practice of medicine. However, there are no instruments to assess it among physicians. Aim: To construct and evaluate the factorial structure and reliability of a questionnaire designed to measure socially responsible behavior in physicians. Material and Methods: The Questionnaire on Medical Socially Responsible Behavior, consisting of 34 items, was constructed. It was applied to 284 physicians and medical students. After eliminating respondents who omitted questions, a valid sample of 214 individuals aged 23 to 67 years (51.4\% males) was obtained. We assessed the factorial structure, reliability, discriminative ability of the items and correlation between factors. Results: Exploratory factorial analysis, conducted using the principal axis method, identified the presence of three factors and considered 30 items. The reliability of the factors, assessed using Cronbach's alpha, ranged from 0.73 to 0.89 . Only one item had a low correlation of 0.3 . Correlations between the three factors were direct and high. Conclusions: The developed questionnaire presents a definite factorial structure, with internally consistent and correlated factors and with adequate psychometric properties.

(Rev Med Chile 2014; 142: 90-97)
\end{abstract}

Key words: Physicians; Psychometrics; Social Responsibility.
E 1 ejercicio de la medicina enfrenta hoy considerables desafíos: cumplir las expectativas de la sociedad en un contexto inseguro y cambiante, donde el médico debe recuperar los valores del profesionalismo, reactualizando el contrato social que tradicionalmente existía y que regulaba los intercambios entre esta profesión y la sociedad $^{1,2}$. En este contexto emerge el concepto de responsabilidad social (RS), que implica el compromiso de los individuos, no sólo con el bienestar propio y de los más cercanos, incluidos quienes requieren los servicios profesionales, sino también con el bienestar de todos, generando equidad para las actuales y futuras generaciones, en el marco de un desarrollo sostenible ${ }^{3,4}$.

El término RS nació asociado a la empresa y su deber con la sociedad, como respuesta a las influencias e impactos que su acción tiene en un mundo globalizado y capitalista, lo cual demanda construir un modelo social de transparencia sobre los impactos de las instituciones en el desarrollo sostenible del planeta ${ }^{5}$. Hitos importantes en el desarrollo de este concepto han sido la declaración del Pacto Mundial de Naciones Unidas 6 , la publicación del Libro Verde de la Unión Europea ${ }^{7}$ y la reciente aparición de la normativa internacional ISO 26000 2010: "Guía de responsabilidad social".

Si bien desde lo organizacional, la universidad 
puede ser considerada una empresa, tiene una función social relevante: formar profesionales, técnicos y ciudadanos, que lideren las transformaciones que el mundo globalizado requiere; además de crear y difundir conocimiento orientado a las necesidades del país. La UNESCO resalta este rol en su "Declaración Mundial sobre la Educación Superior en el siglo XXI: Visión y Acción", que señala: "La educación superior debe reforzar sus funciones de servicio a la sociedad, y, más concretamente, sus actividades encaminadas a erradicar la pobreza, la intolerancia, la violencia, el analfabetismo, el hambre, el deterioro del medio ambiente y las enfermedades..." (artículo 6 b) ${ }^{9}$. Para ser socialmente responsable (SR), la universidad debe desplegar principios y valores por medio de cuatro procesos claves: Gestión, Docencia, Investigación y Extensión ${ }^{10,11}$, cumpliendo así con formar ciudadanos dedicados al progreso social ${ }^{12}$, que interioricen valores como la tolerancia, respeto a los derechos, responsabilización por las propias decisiones y solidaridad ${ }^{13}$.

Para contar con médicos que posean tales atributos, las universidades, y en particular las facultades de medicina, deben desarrollarse como organizaciones SR, y transmitir estos valores a sus estudiantes.

El comportamiento SR se refiere a conductas morales, que tienen a la base una intención orientada hacia el bienestar de todos y que se ejercen con determinada frecuencia; se caracterizan por su carácter prosocial, cooperativo, de participación y de autocontrol ${ }^{14,15}$. La conducta prosocial es intencionada y propositiva, se define siempre en función de las consecuencias que tenga sobre los demás, alude a comportamientos beneficiosos para el otro ${ }^{16}$, y está mediada principalmente por la empatía ${ }^{15}$.

El comportamiento moral requiere componentes derivados de la inteligencia intrapersonal e interpersonal ${ }^{17}$, así como aspectos motivacionales $\mathrm{y}$ cognitivos ${ }^{18}$. Entre estos últimos, Piaget y Kohlberg ${ }^{18,19}$ postularon que los individuos avanzan gradualmente por diversas etapas de desarrollo moral, ligadas al desarrollo cognitivo. Kohlberg definió tres niveles de desarrollo moral (preconvencional, convencional y postconvencional), siendo el tercero de ellos el vinculado a principios morales autónomos y ética universal. Narváez plantea la existencia de tres éticas fundamentales: de la seguridad, de la conexión y de la imagina- ción, la cual tiene un mayor sentido moral que las anteriores, pues busca formas creativas de pensar y actuar socialmente, siendo homologable a la ética de la RS ${ }^{20}$.

La RS es proporcional al alcance del rol de quien lo ejerce; así, la RS del médico sería mayor dado el impacto social de sus decisiones ${ }^{14}$. Pese a esto, la RS en medicina ha sido escasamente tratada, abordándose mayoritariamente desde conceptos como el profesionalismo ${ }^{21}$, potenciado en las últimas décadas por parte de organismos ligados al ejercicio y la formación médica ${ }^{22}$. Asimismo, se ha planteado como un deber de las escuelas de medicina enseñar a sus alumnos a ser $\mathrm{SR}^{23}$. Para esto, las facultades tienen la obligación de dirigir sus actividades educativas, investigadoras y de servicio a atender las necesidades de la comunidad, región o nación que les ha conferido el mandato de servir, siguiendo principios humanísticos que constituyen un marco referencial para la RS: calidad, equidad, relevancia y efectividad ${ }^{24}$. Un hito importante para el futuro de la educación médica en el mundo es el Consenso Global sobre Responsabilidad Social de las Facultades de Medicina del año 2010, donde se plantearon las directrices para que éstas se transformen en $\mathrm{SR}^{25}$.

Hasta el momento, se han generado instrumentos para evaluar el comportamiento SR académico en estudiantes universitarios, como el Cuestionario de Autoatribución de Comportamientos Socialmente Responsables ${ }^{26}$ que ha sido aplicado en estudiantes chilenos, mostrando baja autoatribución de comportamientos $\mathrm{SR}^{27}$. Respecto a la RS profesional, en la Universidad de Concepción se han definido perfiles de profesional SR para algunas carreras de la salud, con posterior incorporación de la RS en sus mallas curriculares ${ }^{28,29}$, no existiendo instrumentos específicos para su medición.

Para los médicos no se dispone de estas definiciones, haciéndose relevante avanzar hacia la generación y validación de instrumentos que contribuyan a la comprensión del ejercicio de comportamientos SR en médicos, y la incorporación del tema en los currículos de la carrera de medicina de las universidades del país.

Esta investigación pretende contribuir a lo anterior, evaluando la validez y confiabilidad de un instrumento para medir este fenómeno: el cuestionario de Comportamientos Médicos Socialmente Responsables (CMSR). 


\section{Método}

Previo a la elaboración del instrumento, se construyó un perfil del médico SR, encuestando a 30 médicos de la provincia de Concepción, que se desempeñaban en diversos ámbitos de la salud, público y privado, solicitándoles que indicaran acciones que caracterizan a un médico SR, lográndose obtener 217 indicadores.

Con el análisis de contenido temático de éstos, se elaboraron 11 categorías, y se seleccionaron 3 indicadores conductuales de cada una, generándose 33 ítems. Con éstos se elaboró un cuestionario de frecuencia de realización de comportamientos SR, el cuestionario CMSR, ante el cual el participante debía evaluar cada indicador según la frecuencia en el propio comportamiento, utilizando una escala tipo Likert de cinco alternativas de frecuencia (desde "Nunca" $=1$ a "Siempre" $=5$ ).

Para evaluar validez de contenido, se recurrió a cuatro jueces expertos en el área, realizándose posteriormente una aplicación piloto a siete médicos. Con las correcciones realizadas se llegó al cuestionario definitivo, de 34 ítems $^{30}$.

La versión final fue aplicada vía electrónica y de manera directa, a médicos de distintos años de ejercicio profesional, lugar y tipo de desempeño, y a un grupo de alumnos de último año de la carrera de medicina de dos universidades de la región. El proceso duró 6 meses, por la dificultad de acceso a la muestra, llegándose a 284 encuestados. La aplicación se hizo previo proceso de consentimiento informado, enfatizándose la confidencialidad y voluntariedad de la participación, y no implicó retribución directa.

Para evaluar la validez de constructo, a partir de la estructura factorial del cuestionario, se utilizó una muestra válida de 214 participantes, luego de eliminar a los sujetos con respuestas omitidas. De ellos, 110 (51,40\%) fueron hombres y 94 (43,93\%) mujeres, con diez casos que no informaron este dato, de edades entre los 23 y 67 años $(M=32,08$; D.E. $=9,95)$. Setenta y cuatro coma siete por ciento de ellos $(n=160)$, provenía de comunas urbanas y $11(5,14 \%)$ de comunas rurales de la Octava Región, 9 de la Región Metropolitana $(4,21 \%)$ y 15 de otras regiones (7\%); un 8,9\% no informó. El nivel formativo se distribuía en 56 alumnos de último año de medicina (26,17\%), 86 médicos generales (40,2\%), y 57 especialistas o médicos en formación de especialidad (26,6\%). Quince sujetos omitieron esta información (7\%). Los 158 médicos tenían entre 0 y 40 años de ejercicio profesional $(\mathrm{M}=6,11$; D.E. $=9,32)$.

Para el análisis estadístico, se evaluó la estructura factorial del cuestionario mediante un Análisis Factorial Exploratorio (AFE), aplicando el método de extracción de eje principal (AEP). Adicionalmente se evaluó la confiabilidad de los factores aplicando el coeficiente Alfa de Cronbach y su correlación mediante coeficiente $r$ de Pearson. Un valor $\mathrm{p}<0,05$ fue considerado estadísticamente significativo. Los análisis se hicieron con el programa estadístico STATA SE 11.0.

\section{Resultados}

La pertinencia de realización del AFE se apoyó en la prueba de adecuación muestral de KáiserMeyer-Olkin (KMO), que fue de 0,88 , y la prueba de esfericidad de Bartlett, que resultó estadísticamente significativa $\chi^{2}(561)=2632,85(\mathrm{p}<0,05)$.

Para identificar el número correcto de factores, considerando los 34 ítems, se usaron cuatro criterios complementarios: el criterio de KaiserGuttman, el criterio de Cattell ${ }^{31}$, el análisis paralelo de Horn, y el test de Minimun Average Partial de Velicer ${ }^{32,33}$. Todos apuntaron a la existencia de tres factores. Se evaluó la organización de los ítems en la estructura trifactorial, mediante AEP, obteniéndose una matriz de configuración, aplicando rotación oblicua Promax. Al obtenerla, se excluyó aquellos ítems con cargas factorial bajo el umbral sugerido por la literatura $(0,30)^{31}$. Para esto se eliminó, entre los ítems bajo el umbral, al que tuviera las cargas factoriales más bajas, y se repitió con el conjunto de ítems remanentes todo el análisis desde el cálculo del KMO en adelante. Así se eliminaron los ítems 4, 19, 8 y 18, sucesivamente.

Con los 30 ítems restantes, se encontró un KMO de 0,89 , con una prueba de esfericidad estadísticamente significativa $\chi^{2}(435)=2344,91$ $(\mathrm{p}<0,001)$, siendo el EFA pertinente. Al estimar el número de factores, el criterio de Kaiser-Guttman identificó tres factores con valores propios (eigenvalues) sobre 1,0, explicando un $85,82 \%$ de la varianza total de los ítems. El criterio de Catell indicó también la presencia de tres factores, Figura 1. El Análisis Paralelo de Horn, en base a 5.000 muestras aleatorias, coincidió en tres factores con valores propios $(8,21 ; 1,71$ y 1,18$)$ sobre los 


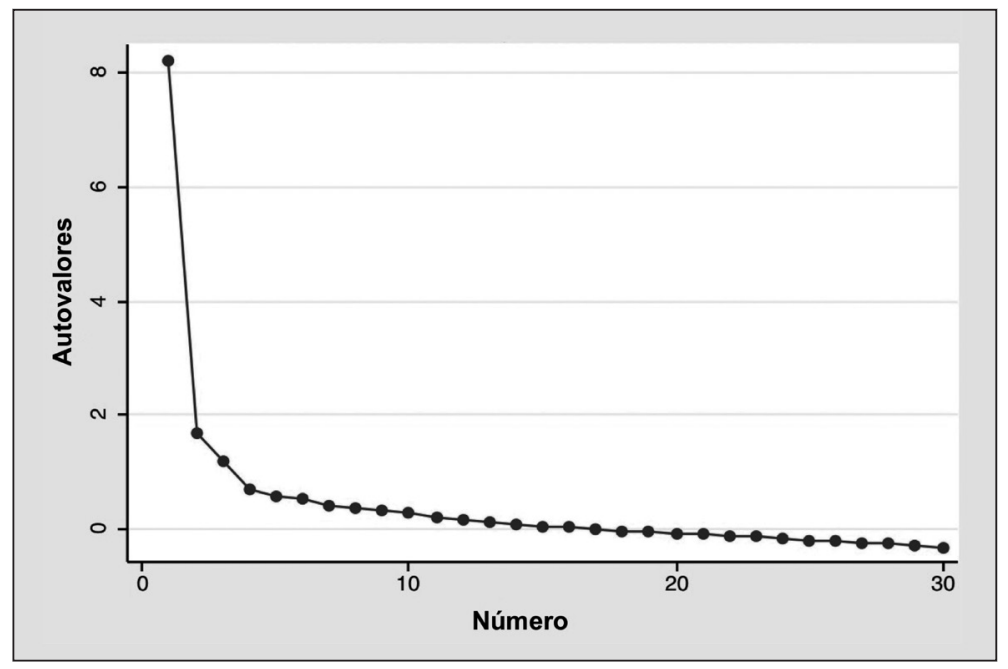

Figura 1. Gráfico de sedimentación para el Cuestionario de Comportamientos Socialmente Responsables (30 ítems). obtenidos en las muestras aleatorias $(1,00 ; 0,85$ y 0,78$)$, al igual que el MAP de Velicer, donde el promedio de los cuadrados de las correlaciones entre los ítems correspondió a 0,08, mientras que el menor de los promedios de los cuadrados de las correlaciones parciales se obtuvo al parcializar el quinto componente, con un promedio de 0,01 .

Se generó, entonces, la matriz de configuración para los tres factores, Tabla 1. Todos los ítems mostraron cargas sobre 0,30 . Sin embargo, el ítem 30 presentó cargas sobre el umbral para dos factores, por lo que su asignación fue sujeta a análisis teórico quedando finalmente adscrita al factor 3 , donde además presentó mayor carga factorial.

Los factores quedaron configurados de la siguiente forma:

Factor 1: Conformado por 15 ítems (32, 24, $23,17,34,33,31,20,25,3,2,16,6,5$ y 7 ), cuyo contenido apuntaba al adecuado cuidado de los pacientes, sus familias y comunidad, denominándose "Orientación al cuidado de los pacientes".

Factor 2: Constituido por 8 ítems $(13,12,10$, $14,15,22,1$ y 11), que aludían al trabajo en equipo, y respeto por el medio ambiente de desempeño, por lo cual se decide denominar como "Orientación al cuidado del entorno laboral".

Factor 3: Incluyó 7 ítems (26,27, 28, 29, 30, 21 y 9), apuntando a "Adecuación a las normas de la profesión".

Al evaluar la confiabilidad, el coeficiente de Cronbach resultó ser $a=0,89$ para el factor
"Orientación al cuidado de los pacientes", con capacidades discriminativas, según la correlación entre los ítems y el total corregido de la escala, entre $r=0,44$ (ítem 7) y $r=0,64$ (ítem 33); $a=$ 0,73 para el factor "Orientación al cuidado del entorno laboral", con correlaciones entre $r=0,26$ (ítem 1, el único bajo 0,30) y $r=0,55$ (ítem 12); y $a=0,75$ para el factor "Adecuación a las normas de la profesión", con correlaciones entre $r=0,33$ (ítem 9) y $r=0,56$ (ítem 27).

Considerando que los factores tenían una confiabilidad adecuada para uso en investigación, se calculó el puntaje de éstos mediante la sumatoria de los códigos asignados a las alternativas de respuesta de cada ítem por factor. Dado que cada factor presenta un número distinto de ítems, se calculó un coeficiente dividiendo la media de cada factor por el número de ítems que lo constituye $(\mathrm{M} / \mathrm{i})$, para facilitar la comparación. Los estadísticos descriptivos de cada factor se exhiben en la Tabla 2, Asimismo, se calculó una prueba de normalidad de los factores indicando asimetría negativa en todos los casos.

Como último punto del análisis psicométrico de los instrumentos, se evaluó la relación entre los factores, utilizando el coeficiente de correlación de Spearman, en base a un contraste unilateral.

Para los tres factores del cuestionario, se encontraron correlaciones directas, estadísticamente significativas y con tamaño del efecto grande (correlaciones intensas) en todos los casos, Tabla 3. 
Tabla 1. Matriz de configuración para el cuestionario de Frecuencia con 30 ítems, obtenida mediante análisis de Eje principal con rotación Promax

\begin{tabular}{|c|c|c|c|c|}
\hline Ítem & $\begin{array}{c}\text { Factor } \\
1\end{array}$ & $\begin{array}{l}\text { Factor } \\
2\end{array}$ & $\begin{array}{c}\text { Factor } \\
3\end{array}$ & $\begin{array}{l}\text { Comuna- } \\
\text { lidades }\end{array}$ \\
\hline $\begin{array}{l}\text { 01. Procurar que al menos una parte de su ejercicio profesional sea en el } \\
\text { Servicio Público }\end{array}$ & & $0,318^{a}$ & & 0,910 \\
\hline $\begin{array}{l}\text { 02. Realizar actividades orientadas a prevenir la aparición de enfermedades en } \\
\text { sus pacientes en su práctica profesional }\end{array}$ & 0,453 & & & 0,735 \\
\hline 03. Promover estilos de vida más sanos en sus pacientes & 0,508 & & & 0,636 \\
\hline $\begin{array}{l}\text { 05. Hacer buen uso de los recursos tanto sectoriales como extrasectoriales, por } \\
\text { ejemplo, usando las redes existentes }\end{array}$ & 0,389 & & & 0,763 \\
\hline $\begin{array}{l}\text { 06. Promover entre los miembros de su equipo de trabajo que se brinde la } \\
\text { mejor atención posible a los pacientes }\end{array}$ & 0,396 & & & 0,706 \\
\hline $\begin{array}{l}\text { 07. Aplicar la mejor evidencia científica disponible para tomar decisiones en } \\
\text { su trabajo }\end{array}$ & 0,338 & & & 0,772 \\
\hline $\begin{array}{l}\text { 09. Derivar oportunamente a los pacientes con problemas de salud que no } \\
\text { puede resolver }\end{array}$ & & & 0,322 & 0,825 \\
\hline $\begin{array}{l}\text { 10. Usar los recursos (electricidad, gas, etc.) y materiales de trabajo (papel, } \\
\text { útiles de oficina), de manera racional en su lugar de trabajo }\end{array}$ & & 0,479 & & 0,666 \\
\hline $\begin{array}{l}\text { 11. Disponer que los desechos de su actividad laboral se eliminen de manera } \\
\text { adecuada }\end{array}$ & & 0,301 & & 0,666 \\
\hline $\begin{array}{l}\text { 12. Cuidar que el entorno de su consulta o lugar de trabajo esté limpio, y libre } \\
\text { de elementos contaminantes (tales como insumos usados en la atención } \\
\text { de pacientes, restos de materiales, etc.) }\end{array}$ & & 0,521 & & 0,575 \\
\hline $\begin{array}{l}\text { 13. Mantener un trato respetuoso con sus compañeros de trabajo, indepen- } \\
\text { dientemente de su profesión }\end{array}$ & & 0,570 & & 0,735 \\
\hline $\begin{array}{l}\text { 14. Promover que sus pacientes recurran a otros profesionales de la salud } \\
\text { cuando lo requieran }\end{array}$ & & 0,477 & & 0,676 \\
\hline 15. Contribuir a generar un clima de trabajo agradable & & 0,432 & & 0,651 \\
\hline $\begin{array}{l}\text { 16. Resolver los problemas de los pacientes trabajando con otros profesionales } \\
\text { de salud }\end{array}$ & 0,445 & & & 0,590 \\
\hline $\begin{array}{l}\text { 17. Cuando atiende pacientes, consultarles por sus preocupaciones, creencias } \\
\text { y el impacto que sus problemas de salud tienen en su vida }\end{array}$ & 0,680 & & & 0,576 \\
\hline $\begin{array}{l}\text { 20. Transmitir sus conocimientos a colegas, alumnos u otros profesionales, } \\
\text { aunque esto no sea parte de sus responsabilidades }\end{array}$ & 0,550 & & & 0,601 \\
\hline $\begin{array}{l}\text { 21. Actuar en beneficio del paciente, evitando daños atribuibles al ejercicio } \\
\text { de la profesión }\end{array}$ & & & 0,369 & 0,769 \\
\hline $\begin{array}{l}\text { 22. Entregar a sus pacientes (y/o sus tutores) toda la información disponible } \\
\text { y a su alcance que ellos deseen conocer sobre su situación, con lenguaje } \\
\text { acorde a su condición y contexto }\end{array}$ & & 0,422 & & 0,587 \\
\hline $\begin{array}{l}\text { 23. Solicitar a los pacientes información sobre aspectos psicológicos y de su } \\
\text { entorno social (familiar, comunitario, laboral) }\end{array}$ & 0,742 & & & 0,403 \\
\hline $\begin{array}{l}\text { 24. Considerar en la atención de los pacientes variables familiares y sociales } \\
\text { que pudieren influir en su salud }\end{array}$ & 0,746 & & & 0,415 \\
\hline $\begin{array}{l}\text { 25. Educar a sus pacientes (y a sus familias si es posible) sobre las condiciones } \\
\text { que afectan su salud }\end{array}$ & 0,528 & & & 0,529 \\
\hline $\begin{array}{l}\text { 26. Cumplir oportunamente con las tareas que se le asignan en su lugar de } \\
\text { trabajo }\end{array}$ & & & 0,631 & 0,558 \\
\hline
\end{tabular}


Responsabilidad social en médicos: cuestionario de comportamientos - D. Alvarado et al

\begin{tabular}{|c|c|c|c|c|}
\hline $\begin{array}{l}\text { 27. Cumplir oportunamente con las responsabilidades administrativas propias } \\
\text { de la institución en que trabaja (registros, procedimientos, notificaciones } \\
\text { y otros) }\end{array}$ & & & 0,617 & 0,534 \\
\hline 28. Cumplir los horarios establecidos en la institución en que trabaja & & & 0,545 & 0,702 \\
\hline 29. Evaluar críticamente su propio desempeño como médico & & & 0,529 & 0,586 \\
\hline $\begin{array}{l}\text { 30. Emprender acciones que le permitan superar sus debilidades en el ejercicio } \\
\text { de su profesión }\end{array}$ & & & 0,440 & 0,534 \\
\hline 31. Realizar actividades de autocuidado (ejercicio, alimentación sana, etc.) & 0,553 & & & 0,670 \\
\hline $\begin{array}{l}\text { 32. Con su ejercicio profesional tratar de contribuir al logro de mejores condi- } \\
\text { ciones de vida en su comunidad }\end{array}$ & 0,760 & & & 0,484 \\
\hline $\begin{array}{l}\text { 33. Con su trabajo tratar de contribuir a la mejora continua de la profesión } \\
\text { médica }\end{array}$ & 0,595 & & & 0,504 \\
\hline $\begin{array}{l}\text { 34. Poner a disposición de la comunidad su aporte como médico, cuando } \\
\text { ésta lo necesita }\end{array}$ & 0,646 & & & 0,551 \\
\hline Autovalores & 8,211 & 1,706 & 1,176 & \\
\hline Varianza explicada & $63,52 \%$ & $13,20 \%$ & $9,10 \%$ & \\
\hline
\end{tabular}

aEn cursiva las cargas superiores a 0,30.

Tabla 2. Estadísticos descriptivos del cuestionario de frecuencia de comportamientos socialmente responsables

\begin{tabular}{|lcccccc|}
\hline & M & D.E. & Mín & Máx & M/i & SK( $\boldsymbol{\chi}^{\mathbf{2})}$ \\
\hline Orientación al cuidado de los pacientes & 61,24 & 7,78 & 37 & 75 & 4,08 & $9,06^{*}$ \\
\hline Orientación al cuidado del entorno laboral & 35,83 & 3,34 & 23 & 40 & 4,48 & $34,77^{* * *}$ \\
\hline Adecuación a las normas de la profesión & 31,48 & 2,81 & 17 & 35 & 4,50 & $34,71^{* * *}$ \\
\hline
\end{tabular}

N = 214; M: media aritmética; D.E.: desviación estándar; Mín: mínimo; Máx: máximo; M/i: producto de la media y el número de ítems; SK $\left(\chi^{2}\right)$ : Ji cuadrado del test de curtosis/asimetría para normalidad; $p<0,05^{*} ; p<0,01 * * ; p<0,001^{* * *}$.

Tabla 3. Correlaciones de Spearman entre los factores Cuestionario de Frecuencia de Comportamientos Socialmente Responsables

\begin{tabular}{|c|c|c|c|}
\hline & 1 & 2 & 3 \\
\hline 1. Orientación al cuidado de los pacientes & - & & \\
\hline 2. Orientación al cuidado del entorno laboral & $0,58 * * *$ & - & \\
\hline 3. Adecuación a las normas de la profesión & $0,50 * * *$ & $0,49 * * *$ & - \\
\hline
\end{tabular}

$N=214 ; p<0,05^{*} ; p<0,01^{* *} ; p<0,001^{* * *}$.

\section{Discusión}

Los resultados muestran que el cuestionario CMSR presenta una estructura trifactorial con factores orientados al cuidado de los pacientes, del ambiente laboral y a la adecuación a las normas de la profesión, con lo cual se reduce la estructura de 11 categorías inicialmente elaborada, sugiriendo que la población encuestada vincula la responsabilidad social principalmente con su ámbito de acción inmediato (pacientes, comunidad, equipo de trabajo, entorno, normativas), omitiendo aspectos de la RS médica relacionados con el compromiso con la sociedad en general y la disminución de las inequidades en salud, que serían subsumidos en los tres factores ya mencionados. 
La consistencia interna obtenida resultó adecuada para su utilización con fines de investigación, y la capacidad discriminativa de los ítems mostró valores pertinentes, demostrando que existe una relación suficiente con el factor al cual pertenecen, excepto en el ítem 1 ("Procurar que al menos una parte de su ejercicio profesional sea en el Servicio Público"), el cual debiera ser revisado o excluido para posteriores análisis psicométricos del cuestionario.

Los factores presentan relaciones directas e intensas entre sí, lo que respalda la idea de que todos son parte del mismo constructo estudiado, la RS médica, por lo cual la orientación al cuidado de los pacientes, al cuidado del entorno laboral y la adecuación a las normas de la profesión son diferenciables pero interdependientes.

No es posible comparar los resultados con otras investigaciones, dada la inexistencia de instrumentos similares que apunten a las particularidades de esta población. No obstante, la contribución de este estudio radica en aportar un perfil conductual del médico SR y un instrumento para evaluar la RS de los médicos, al cual se entrega evidencia de validez de constructo, en términos de su estructura factorial, y de su confiabilidad, demostrando que es apto para emplearse en nuevas investigaciones en este tema.

Esto, aunque su interpretación debe considerar el posible sesgo de deseabilidad social vinculado al tema abordado, que se evidenciaría en la asimetría de la escala.

El instrumento puede ser utilizado en futuras investigaciones, que relacionen el CMSR con aspectos como valores, orientación social, género, satisfacción laboral, institución formadora, o evaluando el impacto de intervenciones curriculares en materia de RS.

Lo anterior es relevante, dada la necesidad de avanzar en el estudio de la RS en médicos, dado el rol que ellos pueden cumplir en avanzar a sociedades más justas y la necesidad de restablecer la confianza que la sociedad ha depositado en estos profesionales.

\section{Referencias}

1. Jovell A. Contrato social y valores en la profesión médica. Rev Adm Sanit 2005; 3 (3): 495-503.

2. Horwitz N. El sentido social del profesionalismo médico. Rev Med Chile 2006; 134: 520-4.
3. Urzúa R. La Responsabilidad Social de las Universidades: Una Guía de Discusión. En: Taller "Elaboración de Estrategia común para la Expansión de la Responsabilidad Social en las Universidades Chilenas". Santiago, octubre 2001.www.participa.cl/wp-contentuploads/2007/10/ lars de la universidadesrurzua.PDF(consultado el 10 de diciembre de 2012).

4. Navarro G. Comportamiento Socialmente Responsable. En: Proyecto Universidad: Construye País. Responsabilidad Social Universitaria, una manera de ser Universidad, teoría y práctica de la experiencia chilena. Santiago, Chile. 2006. p. 79-97. Disponible en http://rsuniversitaria. org/web/images/stories/memoria/UCP\%202006.pdf [consultado el 12 de diciembre de 2012].

5. Comisión Mundial sobre el Medio Ambiente y el Desarrollo. Nuestro Futuro Común. Disponible en http:// www.oarsoaldea.net/agenda21/files/Nuestro\%20futuro\%20comun.pdf[Consultado el 12 de diciembre de 2012].

6. Naciones Unidas. El pacto mundial. Disponible en http://www.un.org/es/globalcompact/ [consultado el 12 de diciembre de 2012].

7. Comisión de las comunidades europeas. Libro Verde. Fomentar un marco europeo para la responsabilidad social de las empresas. Bruselas, 2001 Disponible en http:// www.jussemper.org/Inicio/Recursos/Actividad\%20 Corporativa/Resources/Libro\%20verde.pdf [ consultado el 14 de diciembre de 2012].

8. International Organization for Standardization. ISO 26000 Social Responsability.Disponible en http://www. iso.org/iso/discovering_iso_26000.pdf [consultado el 14 de diciembre de 2012].

9. Organización de las Naciones Unidas para la Educación, la Ciencia y la Cultura (UNESCO). Conferencia Mundial sobre Educación Superior. La educación Superior en el siglo XXI. Visión y Acción. Paris, 1998. Disponible en http://unesdoc.unesco.org/images/0011/ 001163/116345s.pdf [consultado el 10 de diciembre de 2012].

10. Vallaeys F. Breve marco teórico de responsabilidad social universitaria [monografía en internet]. Universidad Internacional. Banco Interamericano de Desarrollo; 2007. Disponible en http://www.rsu.uninter.edu.mx/ doc/marco_conceptual/BreveMarcoTeodelaResponsabilidadSocialUniv.pdf [consultado el 10 de diciembre de 2012].

11. Equipo Coordinador Universidad Construye País. Universidad Construye País. Estrategia y Metodología de intervención para expandir el concepto y la práctica de la Responsabilidad Social Universitaria en Chile. En: Proyecto Universidad: Construye País. Responsabilidad 
Social Universitaria, una manera de ser Universidad, teoría y práctica de la experiencia chilena. Santiago, Chile, 2006. p. 17-39. Disponible en http://rsuniversitaria.org/ web/images/stories/memoria/UCP\%202006.pdf [consultado el 12 de diciembre de 2012].

12. Delors J. Formar a los protagonistas del futuro. Extracto de El Correo de la UNESCO, Abril 1996. Disponible en http://www.unesco.org/education/nfsunesco/pdf/ DELOR2_S.PDF15/12/12

13. Urzúa R, de Puelles M. Educación, gobernabilidad democrática y gobernabilidad de los sistemas educativos. Revista Iberoamericana de Educación 1996; No 12, p. 107-35.

14. Navarro G. Moralidad y Responsabilidad Social: Bases para su desarrollo y educación. Concepción: Universidad de Concepción; 2012.

15. Martí M. Razonamiento Moral y prosocialidad: fundamentos. Madrid: Ed. CCS; 2010.

16. Vander J. Manual de Psicología Social. Barcelona: Paidós; 1986.

17. Gardner H. Inteligencias múltiples. La teoría en la práctica. Barcelona: Ed. Paidós Ibérica; 2005.

18. Papalia D, Wendkos S, Duskin R. Desarrollo Humano. $11^{\circ}$ ed. México D.F.: McGraw-Hill/Interamericana Editores; 2009.

19. Hersch R, Reimer J, Paolito D. El crecimiento moral. De Piaget a Kholberg. Madrid: Ediciones Narcea, S.A.; 1984.

20. Narváez D. La teoría de la ética triuna: premisas básicas e implicaciones. Postconvencionales 2010; 2 (agosto) 74-96.

21. Pardell H. El nuevo profesionalismo médico. Una ideología expresada en conductas. Barcelona: Fundación Medicina y Humanidades Médicas; 2004 Disponible en http://ehu.es/SEMDE/archivos_pdf/humanitas_7. pdf $\#$ page $=19$ [consultado el 17 de diciembre 2012] .

22. Federación Europea de Medicina Interna, el American College of Physicians-American Society ofInternal Medicine (ACP-ASIM) y el American Board of Internal Medicine (declaración conjunta). Profesionalismo médico en el nuevo milenio: una declaración para el ejercicio de la medicina. Ann Intern Med 2002; 136: 243-6.
23. Faulkner L, McCurdy R. Teaching Medical Students Social Responsibility: The Right Thing to Do. Acad Med 2000; 75 (4): p 346-50.

24. Boelen C. Responsabilidad social y excelencia. Educ Med 2009; 12 (4): 199-205.

25. Organización Mundial de la Salud, Universidad de British Columbia, Escuela de Medicina de la Universidad Walter Sisulu. Consenso Global sobre la Responsabilidad Social de las Facultades de Medicina. East London, octubre 2010. Disponible en http://healthsocialaccountability.sites.olt.ubc.ca/files/2011/06/11-06-03-SPANISH-pdfstyle.pdf. [Consultado el 18 de diciembre de 2012].

26. Davidovich M, Espina A, Navarro G, Salazar L. Construcción y estudio piloto de un cuestionario para evaluar comportamientos socialmente. Revista de Psicología de la Universidad de Chile 2005; 14 (1): 125-40.

27. Navarro G, Boero P, Jiménez G. Universitarios y responsabilidad social. Calidad en la Educación 2010; 33 (diciembre): 101-21.

28. Programa de Estudios sobre la Responsabilidad Social. Tercer Reporte de Sustentabilidad Universidad de Concepción. Concepción: Universidad de Concepción. Vicerrectoría de Asuntos Económicos y Administrativos, 2011. Disponible en http://www2.udec.cl/rsu/images/ stories/doc/reporte_3.pdf [consultado el 23 de diciembre de 2012].

29. Rivera N, Rocha F, Troncoso M, González O, Torrico J, Muñoz R, et al. Aplicación de un modelo educativo para la Educación en Responsabilidad Social Profesional a estudiantes del área de la salud, Universidad de Concepción. Rev Educ Cienc Salud 2010; 7 (2): 119-27.

30. Alvarado D, Pérez C, Navarro G. Levantamiento de indicadores observables en la responsabilidad social del ejercicio médico. Rev Educ Cienc Salud. Aceptado a publicación.

31. Hair JF, Black WX, Babin BJ, Anderson RE, Tatham RL. Análisis multivariante. Prentice Hall 2005.

32. Tabachnick BG, Fidell LS. Using multivariate statistics. Boston Allyn and Bacon 2006.

33. Buja A, Eyuboglu N. Remarks on parallel analysis. Multivariate Behav Res 1992; 27: 509-40. 